\title{
15 \\ Towards an Intelligent Software Maintenance Network
}

\author{
P. A. Bailes \\ P. Burnim \\ M. Chapman \\ J. V. Harrison \\ I. Peake \\ Centre for Software Maintenance, Department of Computer Science \\ The University of Queensland QLD 4072, AUSTRALIA \\ Tel: +61733653168 \\ Fax: +61733651999 \\ E-mail:paul@cs.uq.edu.au
}

\begin{abstract}
The extreme difficulty of software maintenance means that specialised support tools are required. There are however potential disadvantages of inaccessibility and non-standard presentations. The presentation problem can be solved by interfacing intelligent maintenance tools to standard presentation environments, such as WWW hypertext browsers. This then poses the question: why not also use WWW technology to make intelligent maintenance tools more accessible?
\end{abstract}

Internet, Maintenance, Reengineering, WWW

\section{Keywords}

\section{INTRODUCTION}

The Intelligent Software Maintenance Network (ISMN) exploits the synergy of Hypertext, with the Internet in order to solve several problems in Software Maintenance. World-WideWeb (WWW) technology is used both as an implementation agent for software maintenance tools, as well as a medium for communicating maintenance information. 


\section{SOFTWARE MAINTENANCE}

What are the special technical requirements and problems posed in order effectively to maintain software?

\subsection{The Nature of Software Maintenance}

Software Maintenance (Pressman, 1992) is to adapt the function of an existing software system for some modified purpose compared to that which it originally performed, combining reverse- and forward engineering processes as follows. In the absence of perfect records of design processes, it is necessary to reverse-engineer design information from existing implementations. Modification to the implementation level takes place in the context of modified design information, enabling more efficient decision-making e.g. about what components of the original implementation are best re-used vs. discarded vs. developed from the beginning.

\subsection{Intelligent Software Maintenance}

Reverse-engineering/design recovery (Chikofsky and Cross, 1990) is thus a key software maintenance process, and represents a supremely-difficult programming problem if it is to be automated. This is because of the general inability of each different programming language to support the direct expression of every design paradigm. Instead, idioms of indirect expression have to be devised by programmers, with the result that the challenge of recognising these idioms lifts the complexity of the undertaking to a level equivalent to that of natural language understanding.

As a result, software reverse-engineering tools used to support software maintenance have to facilitate the application of the maximum amount of intelligence to the design recovery problem, with specific technical implications for reverse-engineering tool development environments as follows (Atkinson et al., 1994).

\section{Persistence}

Foremost, it should be possible to augment programmed (machine) intelligence with human intelligence, as exemplified by the support for human-computer symbiosis in related advanced problem-solving tools (e.g. theorem-provers). The technical prerequisite is to use a persistent store for the intermediate ("internal") results of software system analysis, so that humancontrolled browsing and modification of the knowledge base is possible. 


\section{Expressiveness}

Design recovery is so difficult that use of relatively low-level metalanguages for tool implementation is particularly inefficient. The facts that the reverse-engineering process is likely to be dominated by human interaction and that design recovery from the one software system is not to be repeated frequently, demand that overall process efficiency be achieved by sacrificing the mechanical efficiency of reverse-engineering tools for the efficiency of their development, i.e. by using expressive metalanguages.

\section{Extensibility}

Finally, because human-computer symbiotic tool use will likely uncover further design idioms, it will be advantageous if the development environment supports extensibility. A very efficient way of achieving this result is by self-implementation.

\subsection{Accessibility Problems in Intelligent Software Maintenance}

There are practical problems in the realisation of the above requirements.

\section{Tool Distribution}

The combination of technical characteristics (expressiveness, persistence, extensibility) that is required to support intelligent software maintenance is found only in a very select group of proprietary software environments, most notably Reasoning Systems Inc.'s “Software Refinery" (Newcomb and Markosian, 1993). Such environments pose the following access problems:

the environments are expensive to acquire

the environments are available on specific hardware platforms

tools developed using the environments require specific execution support environments, involving further expense

user modification to tools developed using the environments requires access to the full development environment, not just the execution environment

modification to tools is best performed by (the limited number of) specialists experienced in the development environment.

\section{Information Presentation}

Likewise, the results of design recovery are better presented in standard presentation paradigms.

- The familiarity that is associated with standardisation (even if merely de facto) is a significant aid to human comprehension. 
- The quantity and sophistication of documentation derived from intelligent design recovery may require further machine processing; non-standard presentations are more likely to require proprietary presentation management tools, recreating some of the above problems found in Tool Distribution.

\section{WWW AS PRESENTATION MEDIUM FOR SOFTWARE MAINTENANCE}

The WWW is a standard means of presenting complex information. It is applicable to design recovery as follows.

\subsection{Hypertext as Documentation Style}

The following are questions that can be asked when attempting to comprehend program source code (e.g. when attempting to infer a design):

1. what is the nature of some used entity?

2. what is a defined entity used for?

3. where else is this entity used?

4. what entity is this entity part of?

It is our hypothesis that the (re-)establishment of logical links between the definitions and uses of entities in a software system is critical to inferring design information. We propose that hypertext links be embedded into program source code to provide just this sort of connectivity.

\subsection{Example}

Figure 1 depicts the result of applying our prototype hyperlinking tool displayed with Netscape. Occurrences of identifiers representing entity uses are transformed into the sources of hyperlinks, and are displayed underlined; occurrences of identifiers representing entity definitions are available as the destinations of hyperlinks; not all definitions are used, and only those that are hyperlink targets are displayed in boldface. Naturally, a small example is given here; the full power of the facility better manifests itself on large amounts of code.

Of course, further refinements are possible (e.g. a chain of hyperlinks from definitions to uses) and will be implemented in due course. 


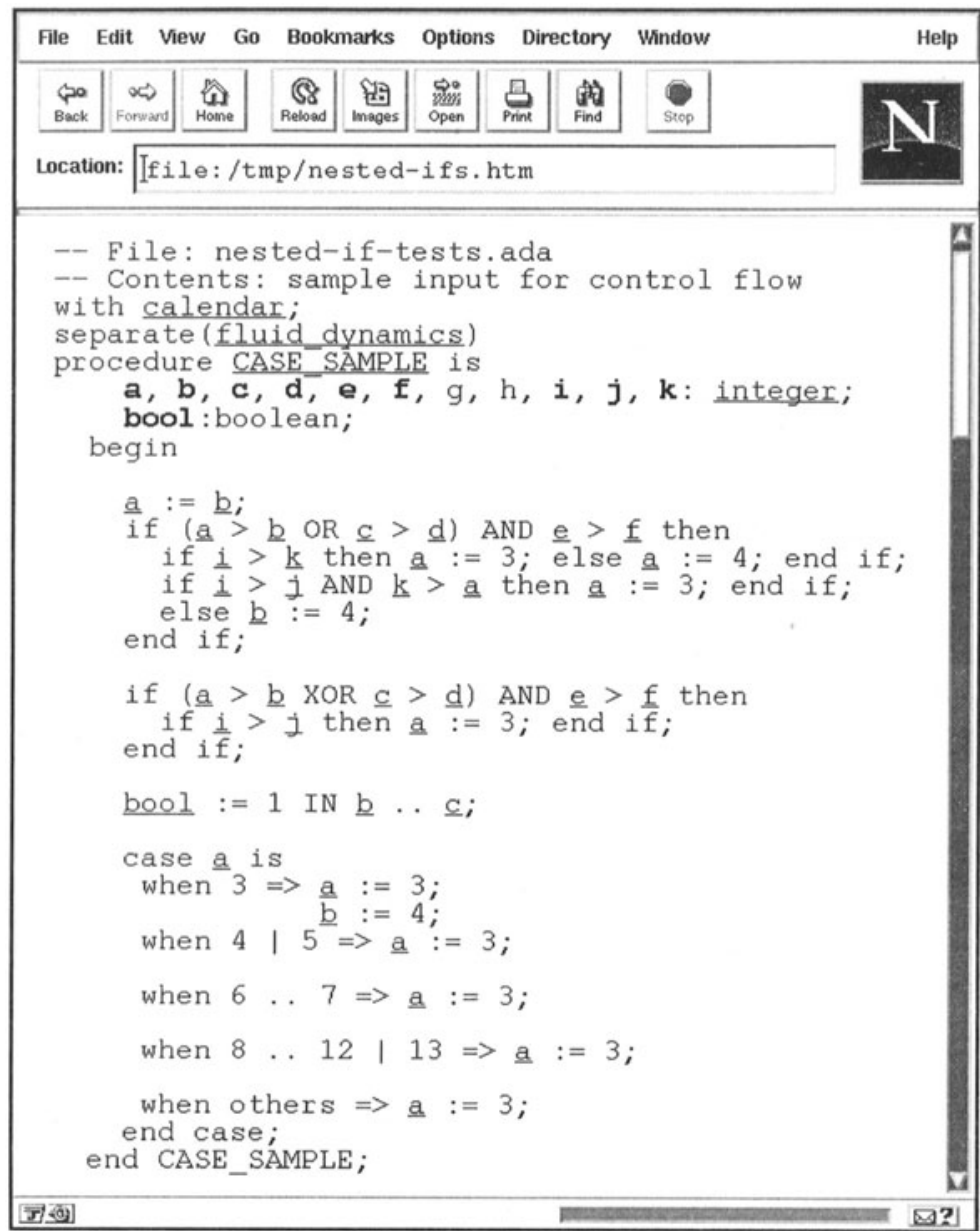

Figure 1 Prototype hyperlinked source code sample.

\subsection{Implementation}

Implementation of the WWW interface for analysed code was a logical and fairly straightforward extension of the existing Ada language model for Software Refinery, available from Reasoning Systems. Their Refine/Ada tool stores an analysed system's parse tree, and allows manipulation and examination of it at a high level. 
Refine/Ada itself matches identifier references with their corresponding definitions, hence all that was required was adding the correct HTML anchors and tags to the source code as follows:

1. definitions that are referenced (ie used): the identifier name is presented in boldface

2. references to non-builtin identifiers: the identifier name is made a hyperlink to identifier's definition.

Further extensions are immediately possible, viz:

1. highlighting (blinking, or italicised) identifiers that are defined but not used

2. for identifier definitions, determining where they are used.

As well as Ada, the we have already developed HTML extensions to C and Ingres/4GL. The code for adding HTML tags has been written in as generic a manner as possible, hence providing this facility for other languages is a small step once a complete language model and linker for the language in question has been created.

\section{INTERNET AS DISTRIBUTION CHANNEL FOR SOFTWARE MAINTENANCE TECHNOLOGY?}

\subsection{Tool Access}

While the ideal operating mode of an intelligent design recovery tool is by a close humancomputer symbiosis, it is sometimes possible to defer the application of human intelligence to a postprocessing phase. For example, in the above the insertion of hypertext links is fullyautomatic, but the inference of design information is both human and subsequent.

Therefore, the logical connection between user and tool need only be a weak one. In may cases batch-mode access to a design recovery tool will suffice. In this case it becomes possible to envisage providing access to a design recovery tool as a straightforward network server, with advantages as follows:

1. batch mode access is more widely-supported than more tightly-coupled access modes, especially across secure firewalls;

2. within the constraints of batch mode, the users are troubled by none of the abovementioned tool distribution problems.

We are currently considering a number of access mechanisms, including

requiring the user to specify the URLs of every source code file 
a forms-based interface through which a user could specify the URL of a directory, from which the server could grab all files with the correct file extension

\subsection{Development Environment Access}

In batch-use mode, user access to the development environment is not required. Indeed, as all tool execution takes place in the domain of the tool developers, opportunities for transmitting the results of tool maintenance and improvement to clients appear significantly enhanced.

\subsection{Compatibility of WWW Presentations}

A further benefit is that WWW provides a common environment for both tool access and information presentation.

\section{FUTURE DIRECTIONS}

The advent of Java (Sun Microsystems, 1995) allows more intelligence to be placed at the client, improving tool access via network. A Java program could be devised which interactively queried the user and the client file system to find and package systems for transmission to the reengineering server. An advanced Java program might even be able to perform some initial naive checks on a system to ensure that all source files are present.

The use of an encryption system such as PGP (Garafinkel, 1995) could provide a secure transport mechanism.

\section{CONCLUSIONS}

Software Maintenance requires intelligence, in both tool implementation and information presentation. WWW technology provides a powerful and standard presentation environment. A virtual strategy of design recovery tool distribution as a networked server overcomes technology access problems. Such ideas have been independently applied previously, in a variety of contexts, e.g. Johnson and Erdem (1995). However, their combination promises to be of considerable additional benefit.

\section{ACKNOWLEDGMENTS}

This work is supported by the Australian Defence Science and Technology Organisation and the Australian Research Council.

"Software Refinery" is a trade mark of Reasoning Systems Inc. 
"Netscape" is a trade mark of Netscape Communications Corporation.

\section{REFERENCES}

Atkinson, S., Bailes, P.A., Chapman, M., Chilvers, M. and Peake, I. (1994) A Re-Engineering Evaluation of Software Refinery ${ }^{\mathrm{TM}}$ : Architecture, Process and Technology, Proceedings 3rd Symposium on Assessment of Quality Software Development Tools, pp. 191200, IEEE.

Chikofsky, E. and Cross, J.H.II (1990) Reverse engineering and design recovery: a taxonomy, IEEE Software, pp. 13-17.

Garafinkel, S. (1995) “PGP: Pretty Good Privacy”, O'Reilly Assocs., Cambridge, Mass.

Johnson, W.L. and Erdem, A. (1995) Interactive Explanation of Software Systems, Proceedings 1995 Knowledge-Based Software Engineering Conference, pp. 155-164, IEEE.

Newcomb, P. and Markosian, L. (1993), Automating the Modularisation of Large COBOL Programs: Application of an Enabling Technology for Reengineering, Proceedings Working Conference on Reverse Engineering, pp. 222-230, IEEE.

Pressman, R.S. (1992) Software Engineering - A Practitioner's Approach (3rd ed.), McGraw Hill, New York.

Sun Microsystems Computer Company (1995) The Java Language Environment: A White Paper.

\section{BIOGRAPHY}

Paul Bailes is Professor and Head of Department of Computer Science at The University of Queensland, from which he obtained his $\mathrm{PhD}$ in Computer Science in 1984. He also directs the Centre for Software Maintenance which he established in 1995 in order to bring together language technologists and domain experts in order to combine their skills in industrial-scale applications.

Paul Burnim is a Senior Research Assistant in the Centre for Software Maintenance, and earned a BSc (Honours) in Computer Science at The University of Queensland in 1991.

Murray Chapman is a Senior Research Assistant in the Centre for Software Maintenance, and earned a BSc (Honours) in Computer Science at The University of Queensland in 1991.

John Harrison is a Lecturer in Computer Science at The University of Queensland, and deputy director of the Centre for Software Maintenance. He received his a $\mathrm{PhD}$ in Computer Science from the University of Arizona in 1992.

Ian Peake is a Research Scholar in the Centre for Software Maintenance, working on a PhD due for completion in 1996. He earned a BSc (Honours) in Computer Science at The University of Queensland in 1991. 